\title{
First-principles calculation of the electron inelastic mean free path in Be metal
}

\author{
V. M. Silkin, ${ }^{1}$ E. V. Chulkov, ${ }^{1,2}$ and P. M. Echenique ${ }^{1,2}$ \\ ${ }^{1}$ Donostia International Physics Center (DIPC) and Centro Mixto CSIC-UPV/EHU, Paseo de Manuel Lardizabal, 4, \\ 20018 San Sebastián/Donostia, Basque Country, Spain \\ ${ }^{2}$ Departamento de Física de Materiales, Facultad de Ciencias Químicas, UPV/EHU, Apdo. 1072, 20080 San Sebastián/Donostia, \\ Basque Country, Spain
}

(Received 14 July 2003; published 21 November 2003)

\begin{abstract}
The Be electron inelastic mean free path (IMFP) is calculated by using the $G W$ approximation of many-body theory. It is demonstrated that the inclusion of band structure effects significantly improves the agreement between the calculated and measured IMFP in the energy range up to $\sim 30 \mathrm{eV}$. We show that the main effect results from the electron group velocity obtained from $a b$ initio band structure calculations, whereas the evaluated linewidth averaged over momenta is not significantly affected with respect to the prediction of a free electron gas model. The comparison of the IMFP computed within two models, namely, the free electron gas model and the full band structure model, supports the idea of the importance of band structure effects for the description of electron transport in this metal for energies below $30 \mathrm{eV}$, and a nearly free-electron-like behavior for excitation energies above $30 \mathrm{eV}$. The calculated plasmon dispersion is in excellent agreement with available experimental data.
\end{abstract}

DOI: 10.1103/PhysRevB.68.205106

PACS number(s): 71.20.Dg, 71.45.Gm, 72.15.Lh

\section{INTRODUCTION}

The electron inelastic mean free path (IMFP) is of great importance for an analysis of data of various spectroscopies such as energy loss spectroscopy, low energy electron diffraction, photoemission spectroscopy, two-photon photoemission, and time-resolved two-photon photoemission, and a lot of experimental and theoretical work on this subject has been done during the last several decades (see, for instance, Refs. 1 and 2). The inelastic mean free path of the electron quasiparticle is defined as a distance that an excited electron covers in the lifetime $\tau$ which sets the duration of the excitation, i.e., the IMFP $\lambda$ is the product of the inelastic lifetime $\tau$ and the group velocity $v$. First calculations of $\tau$ and $\lambda$ were performed by Quinn and Ferrell ${ }^{3}$ in a degenerate electron gas model within the self-energy formalism of manybody theory. Later these evaluations were extended to include, within the first-Born and random-phase approximations, energies far away from the Fermi surface. ${ }^{4,5}$ Since then, many calculations of the IMFP and electronelectron scattering rates (inverse lifetimes) have been performed with the inclusion of exchange and correlation effects, ${ }^{6-9}$ chemical potential renormalization, ${ }^{10,11}$ plasmon damping, ${ }^{12}$ and core polarizability. ${ }^{13}$ Statistical approximations were also used in the evaluations of the IMFP. ${ }^{14,15}$ Band structure effects, including spin dependence of the IMFP, were discussed in Refs. 16-20. Very recently more sophisticated methods, namely, first-principles band structure methods, have been used to perform ab initio calculations of the inverse lifetime (linewidth) for low energy hot electrons and holes in metals ${ }^{21-27}$ and in a large energy interval for silicon. ${ }^{28}$ The ab initio $G W$ evaluations of the IMFP at high energies have also been performed for beryllium chalcogenides. ${ }^{29}$ As a result of these intensive studies it is believed now that the behavior of the IMFP for high energies (higher than several tens of electronvolts) is well understood and well described in the framework of existing theories. ${ }^{1,2}$
For example, a theoretical prediction is that for high energies the IMFP in free-electron-like metals is a function of the valence electron density only and has a similar behavior. However, there are indications that even for energies above $30 \mathrm{eV}$ the band structure of some materials is far from nearly-free-electron-like. ${ }^{30}$ At low energies the IMFP is very sensitive to details of the band structure. ${ }^{21-26}$ In particular, the deviations of the IMFP from the free-electron-like prediction at energies around $10 \mathrm{eV}$ found for $\mathrm{K}$ and $\mathrm{Rb}$ (Ref. 31 ) were attributed to unoccupied $d$ bands which are situated at these energies. ${ }^{27,31}$ But even more profound discrepancies between the experimental and theoretical IMFPs were found for beryllium. ${ }^{32}$ Photoemission measurements of Johansson and Sernelius ${ }^{32}$ showed that the experimental IMFP, $\lambda$, has a minimum for energies close to $25 \mathrm{eV}$, going down to $1.8 \AA$. The calculations performed within the degenerate free electron gas (FEG) model do not reproduce this minimum. Moreover these evaluations place the theoretical $\lambda(E)$ curve higher than the experimental one by a factor of 3-4 for excitation energies below $30 \mathrm{eV}^{32}$ The authors of Ref. 32 proposed several possible explanations of the observed discrepancies between their experimental results and the FEG theory, in particular the following.

(1) Flat or negative dispersion of a bulk plasmon in a real crystal can significantly reduce the measured IMFP.

(2) The experimental IMFP may be reduced by excitation of surface plasmon.

(3) The reduction of the IMFP is produced by excitation of surface states, which play important role in the surfaces of Be. ${ }^{33-36}$

The two last explanations were motivated by the use of the measurement method: the authors of Ref. 32 derived the inelastic mean free path from the measured core level shifts of $\mathrm{Be}$ atoms in two surface layers of $\mathrm{Be}(0001)$ and $\mathrm{Be}(10 \overline{1} 0)$ by using the layer attenuation model. ${ }^{31}$ These core level shifts can be affected, in principle, by a surface collective excitation (surface plasmon) as well as by a single-particle 
excitation from the $\bar{\Gamma}$ surface state band on $\operatorname{Be}(0001)$ (Refs. 33 and 34$)$ or from the $\bar{A}$ surface state on $\operatorname{Be}(10 \overline{1} 0) .{ }^{35}$ In the present work we show that, in fact, most of the discrepancies between the experiment and the calculation based on the FEG model, ${ }^{32}$ may be removed by taking explicitly into account the Be bulk band structure. We investigate the plasmon dispersion in bulk Be and demonstrate that although it deviates from the prediction of the FEG model, it does not influence significantly the IMFP in beryllium derived from the FEG model. We demonstrate that the main effect of the band structure on the IMFP comes from the fact that the group velocity in beryllium is significantly lower than that evaluated within the FEG model, thus leading to much better agreement with the experiment. On base of the performed calculations of both the electron lifetime and the group velocity we analize relative importance of band structure effects on IMFP in Be for two energy regions, namely, below and above $30 \mathrm{eV}$.

The paper is organized as follows. In Sec. II we give the brief description of the self-energy approach of many-body theory for the evaluation of the inverse lifetime (inelastic linewidth of an excited electron). The calculation results are presented and discussed in Sec. III and the conclusions are given in Sec. IV. Unless otherwise stated, atomic units are used throughout, i.e., $e^{2}=\hbar=m_{e}=1$.

\section{CALCULATION METHOD}

Band structure calculations yield one-electron states, which are characterized by energies $\varepsilon_{n \mathbf{k}}$ and wave functions $\psi_{n \mathbf{k}}(\mathbf{r})$, where $n$ and $\mathbf{k}$ are the band number and momentum, respectively. To all these one-electron states one can attribute a group velocity $\vec{v}_{n \mathbf{k}}$ defined as ${ }^{37}$

$$
\vec{v}_{n \mathbf{k}}=\partial \varepsilon_{n \mathbf{k}} / \partial \mathbf{k}
$$

and the lifetime $\tau_{n \mathbf{k}}$. By using the Heisenberg relation $\tau_{n \mathbf{k}} \Gamma_{n \mathbf{k}}=1$ the linewidth $\Gamma_{n \mathbf{k}}$ of a given electronic state is determined as the inverse lifetime $\tau_{n \mathbf{k}}^{-1}$. Knowing $\vec{v}_{n \mathbf{k}}$ and $\Gamma_{n \mathbf{k}}$, we calculate the inelastic electron mean free path $\vec{\lambda}_{n \mathbf{k}}$ of a given state as

$$
\vec{\lambda}_{n \mathbf{k}}=\vec{v}_{n \mathbf{k}} / \Gamma_{n \mathbf{k}} .
$$

In the FEG model, where a simple relation between the momentum $\mathbf{k}$ and the one-electron energy $\varepsilon_{n \mathbf{k}}=\mathbf{k}^{2} / 2 m$ exists, the group velocity $\vec{v}_{n \mathbf{k}}$ and linewidth $\Gamma_{n \mathbf{k}}$ are functions of an absolute value of momentum; i.e., they depend only on a quasiparticle energy, while band structure based theories give the velocity and the electron linewidth as functions of both the energy and momentum. For a comparison with experimental results, one should evaluate $\lambda$ resolved in energy only. The electron IMFP in bulk Be was obtained in Ref. 32 from measurements of core-level shifts in the first two atomic layers. In such types of experiment, after an excitation from the core-level shells, which form bands with a zero dispersion, excited electrons with the same energy have similar probabilities, in principle, to occupy all one-electron states characterized by different momenta $\mathbf{k}$. Therefore, in

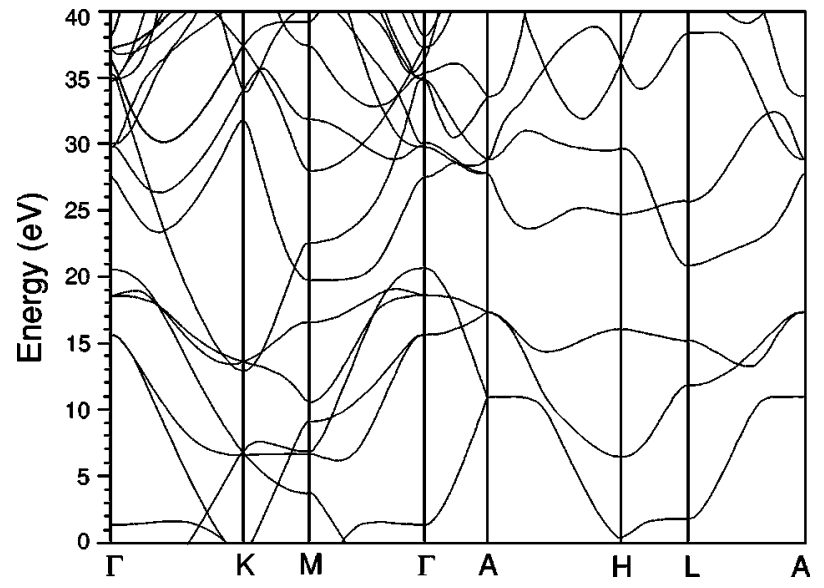

FIG. 1. The calculated unoccupied electronic structure of Be along symmetry directions of the BZ. The zero energy value corresponds to the Fermi level.

the present work we assume an equal probability for population of one-electron states with different $\mathbf{k}$ for the same energy $\varepsilon$. With this assumption we evaluate values of the IMFP, $\lambda_{n \mathbf{k}}$ as a modulus of vector quantity, $\lambda_{n \mathbf{k}}=\left|\vec{\lambda}_{n \mathbf{k}}\right|$. Then, for comparison with experiment, we determine IMFP $\lambda(\varepsilon)$ as an average of $\lambda_{n \mathbf{k}}$ over all wave vectors and energy bands with the same excitation energy in the irreducible part of the Brillouin zone (IBZ). After this procedure one obtains the value of the mean free path $\lambda(\varepsilon)$ as a function of electron energy $\varepsilon$ only.

For the evaluation of wave functions $\psi_{n \mathbf{k}}(\mathbf{r})$ and oneelectron energies $\varepsilon_{n \mathbf{k}}$ we employ a self-consistent pseudopotential method with the local-density approximation (LDA) for the exchange-correlation potential. ${ }^{38}$ To describe the Be electron-ion interaction, a norm-conserving pseudopotential $^{39}$ has been used. The calculations have been performed for a hcp crystal structure with experimental lattice parameters $a=2.2850 \AA$ and $c=3.5847 \AA .{ }^{40}$ In the wave function expansion the plane waves up to $E_{\text {cut }}=24$ Ry have been included. The corresponding unoccupied band structure along high symmetry directions of the Brillouin zone is shown in Fig. 1.

The linewidth $\Gamma_{n \mathbf{k}}$ of the electron state is related to the imaginary part of the electron self-energy $\Sigma_{n \mathbf{k}}:{ }^{41}$

$$
\Gamma_{n \mathbf{k}}=-2 \operatorname{Im} \Sigma_{n \mathbf{k}},
$$

where $\operatorname{Im} \Sigma_{n \mathbf{k}}$ is evaluated as a projection of $\operatorname{Im} \Sigma\left(\mathbf{r}, \mathbf{r}^{\prime} ; \varepsilon_{n \mathbf{k}}\right)$ onto the one-electron state $\psi_{n \mathbf{k}}(\mathbf{r}),{ }^{42,43}$

$$
\operatorname{Im} \Sigma_{n \mathbf{k}}=\int \psi_{n \mathbf{k}}^{*}(\mathbf{r}) \operatorname{Im} \Sigma\left(\mathbf{r}, \mathbf{r}^{\prime} ; \varepsilon_{n \mathbf{k}}\right) \psi_{n \mathbf{k}}\left(\mathbf{r}^{\prime}\right) \mathrm{d} \mathbf{r} \mathrm{d} \mathbf{r}^{\prime}
$$

The self-energy $\Sigma\left(\mathbf{r}, \mathbf{r}^{\prime} ; \varepsilon_{n \mathbf{k}}\right)$ is calculated within the $G W$ approximation of many-body theory, ${ }^{42}$ with $W$ being the dynamically screened Coulomb interaction, and the Green function $G$ replaced by the noninteracting Green function. Thus, for $\operatorname{Im} \Sigma$, one obtains 


$$
\begin{aligned}
\operatorname{Im} \sum & \left(\mathbf{r}, \mathbf{r}^{\prime} ; \varepsilon_{n \mathbf{k}}\right) \\
= & \sum_{m, \mathbf{q}}^{\varepsilon_{F}<\varepsilon_{m \mathbf{q}}<\varepsilon_{n \mathbf{k}}} \psi_{m \mathbf{q}}^{*}(\mathbf{r}) \operatorname{Im} W\left(\mathbf{r}, \mathbf{r}^{\prime} ; \Delta E_{n \mathbf{k}, m \mathbf{q}}\right) \psi_{m \mathbf{q}}\left(\mathbf{r}^{\prime}\right),
\end{aligned}
$$

where the sum is extended over the final states $\psi_{m \mathbf{q}}(\mathbf{r})$ with energy $\varepsilon_{m \mathbf{q}}, \varepsilon_{F}$ is the Fermi energy, and $\Delta E_{n \mathbf{k}, m \mathbf{q}}=\varepsilon_{n \mathbf{k}}$ $-\varepsilon_{m \mathbf{q}}$. The screened Coulomb interaction $W\left(\mathbf{r}, \mathbf{r}^{\prime}, \omega\right)$ is

$$
W\left(\mathbf{r}, \mathbf{r}^{\prime} ; \omega\right)=\int \epsilon^{-1}\left(\mathbf{r}, \mathbf{r}^{\prime \prime} ; \omega\right) V\left(\mathbf{r}^{\prime \prime}-\mathbf{r}^{\prime}\right) \mathrm{d} \mathbf{r}^{\prime \prime} .
$$

Here, $\epsilon^{-1}\left(\mathbf{r}, \mathbf{r}^{\prime \prime}, \omega\right)$ and $V(\mathbf{r})$ represent the inverse dielectric function and the bare Coulomb potential, respectively. Within the random phase approximation (RPA) the Fourier transform of the dielectric matrix $\epsilon_{\mathbf{G G}^{\prime}}(\mathbf{k}, \omega)$, where $\mathbf{G}$ and $\mathbf{G}^{\prime}$ are reciprocal lattice vectors, is related to the densitydensity response function $\chi_{\mathbf{G G}^{\prime}}^{0}(\mathbf{k}, \omega)$ for a noninteracting electron system,

$$
\epsilon_{\mathbf{G G}^{\prime}}(\mathbf{k}, \omega)=\delta_{\mathbf{G G}^{\prime}}-\chi_{\mathbf{G G}^{\prime}}^{0}(\mathbf{k}, \omega) V_{\mathbf{G}^{\prime}}(\mathbf{k}),
$$

where $V_{\mathbf{G}}(\mathbf{k})$ is the Fourier transform of the bare Coulomb potential:

$$
V_{\mathbf{G}}(\mathbf{k})=\frac{4 \pi}{|\mathbf{k}+\mathbf{G}|^{2}}
$$

and

$$
\begin{aligned}
\chi_{\mathbf{G G}^{\prime}}^{0}(\mathbf{k}, \omega)= & \frac{2}{\Omega} \sum_{\mathbf{q}}^{B Z} \sum_{n, n^{\prime}} \frac{f_{n \mathbf{q}}-f_{n^{\prime} \mathbf{k}+\mathbf{q}}}{\varepsilon_{n \mathbf{q}}-\varepsilon_{n^{\prime} \mathbf{k}+\mathbf{q}}+(\omega+\mathrm{i} \eta)} \\
& \times\left\langle\psi_{n \mathbf{q}}\left|e^{-\mathrm{i}(\mathbf{k}+\mathbf{G}) \cdot \mathbf{r}}\right| \psi_{n^{\prime} \mathbf{k}+\mathbf{q}}\right\rangle \\
& \times\left\langle\psi_{n^{\prime} \mathbf{k}+\mathbf{q}}\left|e^{\mathrm{i}\left(\mathbf{k}+\mathbf{G}^{\prime}\right) \cdot \mathbf{r}}\right| \psi_{n \mathbf{q}}\right\rangle .
\end{aligned}
$$

In Eq. (9) the sums run over the q vectors in the first Brillouin zone and over all bands $n$ and $n^{\prime} . f_{n \mathbf{q}}$ is the Fermi distribution function, $\eta$ a positive infinitesimal, and $\Omega$ the crystal volume.

As it has been shown by Maddocks et al., ${ }^{44}$ the RPA is a good approximation for calculation of $\epsilon_{\mathbf{G G}^{\prime}}^{-1}(\mathbf{q}, \omega)$ for $\mathrm{Be}$, that is a key quantity for evaluation of $\Gamma_{n \mathbf{k}}$. Therefore, in the present work we perform calculations within the RPA.

To evaluate the density-density response function $\chi^{0}$, a method of analytical continuation similar to that used in Ref. 44 has been employed. A sampling over the BZ was performed on the mesh of $6912 \mathbf{q}$ points. For electron excitation energies under study (below $50 \mathrm{eV}$ ), the inclusion of up to 50 energy bands in Eq. (9) has been required.

Using Fourier transforms for all quantities in Eqs. (4) and (5) we obtain

$$
\begin{aligned}
\Gamma_{n \mathbf{k}}= & 2 \sum_{m \mathbf{q}}^{\varepsilon_{F}<\varepsilon_{m \mathbf{q}}<\varepsilon_{n \mathbf{k}}} \sum_{\mathbf{G}, \mathbf{G}^{\prime}} \operatorname{Im} W_{\mathbf{G G}^{\prime}}\left(\mathbf{k}-\mathbf{q}, \varepsilon_{n \mathbf{k}}-\varepsilon_{m \mathbf{q}}\right) \\
& \times B_{n m}^{*}(\mathbf{k}, \mathbf{q} ; \mathbf{G}) B_{n m}\left(\mathbf{k}, \mathbf{q} ; \mathbf{G}^{\prime}\right),
\end{aligned}
$$

where $W_{\mathbf{G G}^{\prime}}$ is the Fourier transform of the screened Coulomb interaction $W\left(\mathbf{r}, \mathbf{r}^{\prime} ; \omega\right)$ and $B_{n m}(\mathbf{k}, \mathbf{q} ; \mathbf{G})$ are

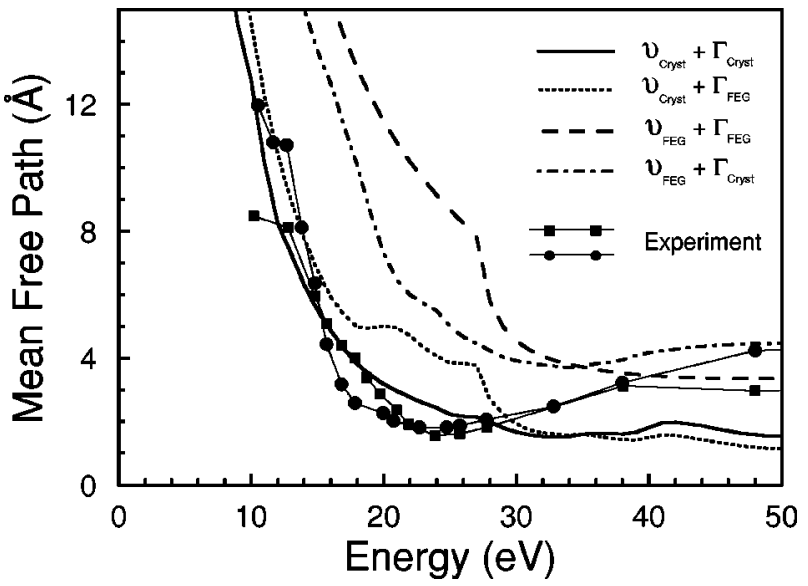

FIG. 2. The IMFP dependence on the electron energy. The solid line shows the calculated ab initio IMFP, and the dashed line corresponds to the IMFP calculated within the FEG model. The dotted line represents the IMFP with the linewidth obtained from the FEG model and the group velocity from real band structure of Be. The dash-dotted line describes the IMFP with the linewidth derived from first principles and the group velocity taken from the FEG model. Filled circles and squares are the experimental values ob-

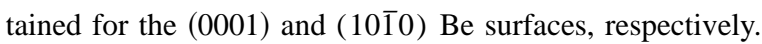

$$
B_{n m}(\mathbf{k}, \mathbf{q} ; \mathbf{G}) \equiv \sum_{\mathbf{G}^{\prime}} a_{n}^{*}\left(\mathbf{k}+\mathbf{G}+\mathbf{G}^{\prime}\right) \cdot a_{m}\left(\mathbf{q}+\mathbf{G}^{\prime}\right),
$$

and $a_{n}(\mathbf{k}+\mathbf{G})$ are the wave function expansion coefficients. In Eq. (10) summing has been performed over $765 \mathbf{q}$ points in the IBZ. To estimate the accuracy which is achieved with this number of $\mathbf{q}$ points we have also performed calculations with 425 q points in the IBZ. The results of both the calculations of $\Gamma_{n \mathbf{k}}$ agree within $1 \%$. On the other hand, $\Gamma_{n \mathbf{k}}$ is more sensitive to the number of the reciprocal lattice vectors $\mathbf{G}$ used in Eq. (10), and final results have been obtained with the use of $40 \mathbf{G}$ vectors. This number ensures a convergence of $\Gamma_{n \mathbf{k}}$ for $\mathbf{k}$ points near the center of the BZ within a few percent. At the same time, for $\mathbf{k}$ near the borders of the BZ the accuracy is $\sim 5 \%$. Taking into account every step of our calculations, we estimate the overall accuracy of the evaluated values of $\Gamma_{n \mathbf{k}}$ around $10 \%$ (of course, any estimations related to the different level of consideration beyond the $G W$ approximation are not included in this analysis).

\section{CALCULATION RESULTS AND DISCUSSION}

In Fig. 2 we compare the evaluated IMFP $\lambda(\varepsilon)$ with experimental data. ${ }^{32}$ To elucidate the role that the group velocity $v$ and the linewidth $\Gamma$ play in the calculation of $\lambda$, in Fig. 2 we show the results of four kinds of our calculations. In the first one (dashed line), both the group velocity and the linewidth were computed within the FEG model. In the second one (dotted line) the FEG model was combined with the first-principles computation results, i.e., the linewidth was obtained from the FEG model and the group velocity from the first-principles results. In the third one (dash-dotted line) the linewidth was derived from the first-principles results and the velocity was taken from the FEG model. Finally, in 


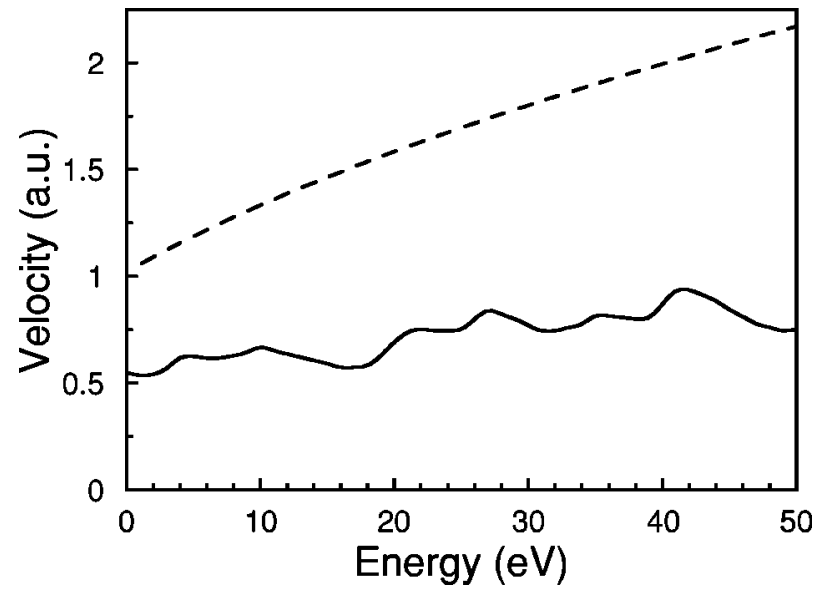

FIG. 3. Group velocities obtained from the ab initio (solid line) and the FEG models (dashed line) calculations.

the fourth kind of calculations (solid line) both $v$ and $\Gamma$ were obtained from the first-principles results. The comparison of the theoretical and experimental results demonstrates that the inclusion of the band structure in the calculation dramatically changes the behavior of $\lambda(\varepsilon)$ obtained within the FEG model, and leads to a good agreement with the experimental data in the energy interval from $\sim 15$ to $\sim 30 \mathrm{eV}$. For excitation energies above $30 \mathrm{eV}$ there are only a few experimental points and a significant scatter between them is observed. ${ }^{32}$ As follows from Fig. 2 the calculated $a b$ initio IMFP for energies above $30 \mathrm{eV}$ is systematically lower than the experimental one, although the difference between the calculated and measured curves is comparable with the accuracy of the experiment.

The major effect of the inclusion of band structure in $\lambda(\varepsilon)$ results from the electron velocity $v{ }^{45}$ In Fig. 3 we show the computed averaged electron group velocity as a function of energy (solid line) and compare it with the free electron group velocity (dashed line) in the energy interval from the Fermi level to $50 \mathrm{eV}$. The figure demonstrates clearly that the averaged band structure group velocity $v_{\text {band }}$ varies slightly around 0.6 a.u. in this energy region while the free electron velocity $v_{\text {free }}$ behaves as $\left(\varepsilon-\varepsilon_{F}\right)^{1 / 2}$, being 1.03 a.u. at the Fermi level $\varepsilon_{F}$. This distinct energy dependence leads to large differences in the velocities between two models starting from a value of $\sim 2$ at $\varepsilon_{F}$ and achieving a value of 3.3 for the ratio $v_{\text {free }} / v_{\text {band }}$.

Figure 4 shows the corresponding impact of band structure on $\Gamma(\varepsilon)$. In this figure the full line represents $\Gamma(\varepsilon)$ obtained from the ab initio calculation and averaged over the $\mathrm{BZ}$ as described above, the dashed line shows $\Gamma(\varepsilon)$ obtained from the FEG model. As follows from a comparison of these two curves, the main effect of the inclusion of the band structure in $\Gamma$ is the increase (decrease) of $\Gamma$ for energies below (above) $34 \mathrm{eV}$. This effect can be attributed to different plasmon dispersions in bulk Be obtained from the first-principles calculations and from the FEG model. In Fig. 5 we show the plasmon dispersion derived from the ab initio calculation of $\epsilon^{-1}(\mathbf{k}, \omega)$ along three high symmetry directions $\Gamma M, \Gamma K$, and $\Gamma A$ of the $\mathrm{BZ}$ and compare it with that obtained from the FEG model and with experimental data. ${ }^{46,47}$ As one can see,

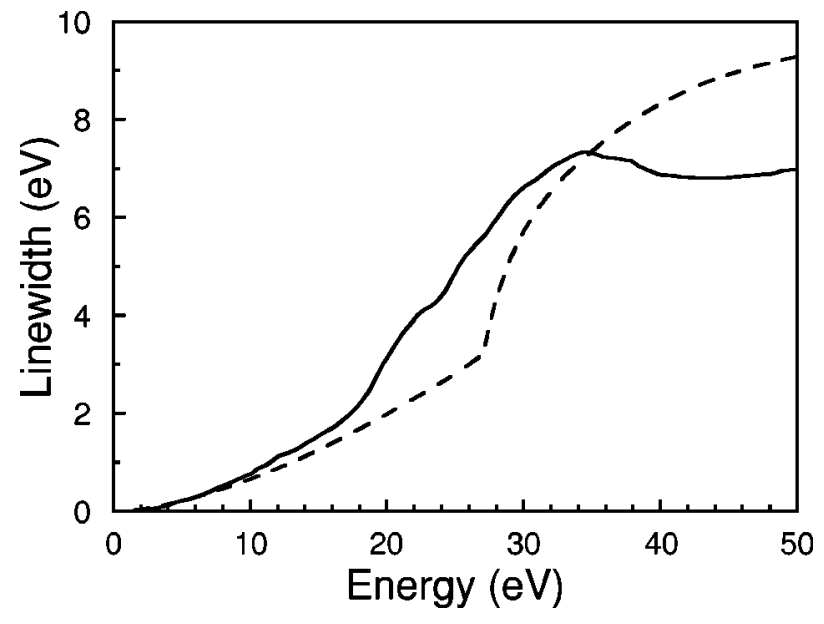

FIG. 4. The calculated linewidth: the solid line shows ab initio results and the dashed line represents the FEG model linewidth.

the first-principles plasmon dispersion goes lower than that of the FEG model. Therefore the first-principles plasmon curves enter the electron gas continuum, where the electronhole excitations may exist, at substantially lower energies. This plasmon dispersion behavior favors the larger ab initio $\Gamma$ compared to the FEG model $\Gamma$. The evaluated firstprinciples bulk plasmon dispersion in $\mathrm{Be}$ is consistent with the experimental results. ${ }^{46}$ Also, the ab initio calculation gives clear the directional anisotropy of the plasmon dispersion observed experimentally: ${ }^{46}$ the dispersion in the $\Gamma-A$ direction (perpendicular to the hexagonal plane) is systematically higher than in the $\Gamma-K$ and $\Gamma-M$ directions (the hexagonal plane) for momenta $q>0.3$ a.u. $^{-1}$. One can note that some discrepancy between the experimental and ab initio dispersions may be ascribed to the difficulty of extracting the plasmon position from the loss energy spectra since in Be the

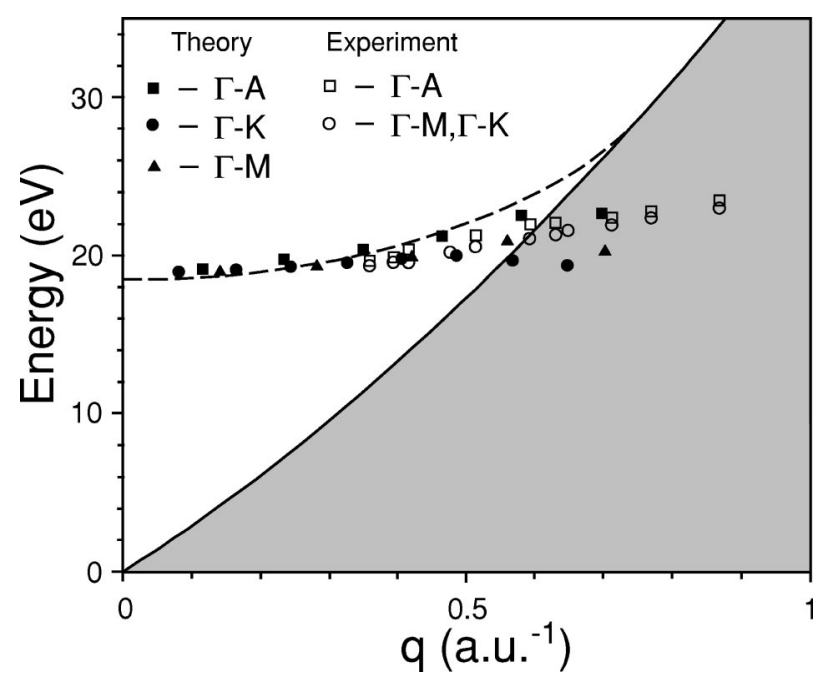

FIG. 5. Plasmon dispersion in Be. The $a b$ initio dispersion is shown by filled squares $(\Gamma-A$ direction), the filled triangle $(\Gamma-M$ direction), and filled circles ( $\Gamma-K$ direction). The FEG model result is shown by the dashed line. Open symbols correspond to the experimental results. The dotted line is the averaged plasmon dispersion obtained from the $a b$ initio calculation. 
plasmon peaks are severely affected by band structure effects and have a linewidth of the order $3-5 \mathrm{eV}^{46}$ Thus one can see that taking into account a crystal structure in the calculation of the self-energy leads to an increase of $\Gamma$ in the energy region below $\sim 30 \mathrm{eV}$ and to a decrease of it for higher energies. This increase of $\Gamma$ for excitation energies below 30 $\mathrm{eV}$ leads to a better agreement with the experiment. However, this effect is of minor importance compared to that introduced by the electron velocities.

Some discrepancy with the experiment for energies higher than $\sim 30 \mathrm{eV}$ can be attributed to more sophisticated calculations of the self-energy and the use of a more relevant group velocity for these energies. As shown by Shung et al. in the self-energy calculations for the FEG model, ${ }^{48}$ the use of a real part of $\Sigma(\varepsilon)$ for $r_{s}=2$ (for $\mathrm{Be} r_{s}=1.866$ ) increases the group velocity of electrons and, consequently, increases the IMFP for $\varepsilon>30 \mathrm{eV}$. On the other hand, Fleszar and Hanke have shown for bulk Si that even in a semiconductor the improved calculation of the real part of the self-energy makes electronic bands more similar to a free electron band for energies above $30 \mathrm{eV}^{28}$ This effect leads to higher group velocities compared to the LDA case. Therefore, one can expect that for $\varepsilon>30 \mathrm{eV}$ the use of the quasiparticle band structure for bulk Be can move up the group velocity curve in Fig. 4, leading to a better agreement with the experiment. The authors of Ref. 28 also showed that the inclusion of vertex corrections in the self-energy can decrease $\operatorname{Im} \Sigma$ for $\varepsilon>30$ thus leading to better agreement between the theoretical IMFP and the experimental one.

\section{CONCLUSION}

In summary, we have performed a first-principles calculation of the IMFP for bulk beryllium within the PRA. A good agreement with the experimental $\lambda$ (Ref. 32) has been obtained for energies from $\sim 15$ to $\sim 30 \mathrm{eV}$. For energies $\varepsilon$ $>30 \mathrm{eV}$ the deviations from the experimental data are comparable with the accuracy of the experiment. We have shown that the inclusion of the group velocity is crucial to account for the experimental IMFP for energies $15<\varepsilon<30 \mathrm{eV}$. The band structure effects in the $\operatorname{Im} \Sigma$, being in general of less importance for $\operatorname{Im} \Sigma$ in this energy interval than for the group velocity, are important to obtain better agreement with the experiment. We have also shown that the calculated plasmon peak dispersion is in good agreement with experimental data. $^{46,47}$

On the basis of the presented results one can interpret the experimental data for the IMFP in $\mathrm{Be},{ }^{32}$ taking into account only bulk properties without invoking any of its surface features. Perhaps the inclusion of the surface properties of Be could lead to an even better agreement with the experiment.

\section{ACKNOWLEDGMENT}

We acknowledge partial support by the University of the Basque Country, the Departamento de Educación del Gobierno Vasco, and the Spanish Ministerio de Educación y Cultura.
${ }^{1}$ C.J. Powell, Surf. Sci. 44, 29 (1974)

${ }^{2}$ C.J. Powell and A. Jablonski, J. Phys. Chem. Ref. Data 28, 19 (1999), and references therein.

${ }^{3}$ J.J. Quinn and R.A. Ferrell, Phys. Rev. 112, 812 (1958).

${ }^{4}$ R.H. Ritchie, Phys. Rev. 114, 644 (1959).

${ }^{5}$ J.J. Quinn, Phys. Rev. 126, 1453 (1962).

${ }^{6}$ R.H. Ritchie and J.C. Ashley, J. Phys. Chem. Solids 26, 1689 (1965).

${ }^{7}$ L. Kleinman, Phys. Rev. B 3, 2982 (1971).

${ }^{8}$ C.A. Kukkonen and A.W. Overhauser, Phys. Rev. 20, 550 (1979).

${ }^{9}$ D.R. Penn, Phys. Rev. B 22, 2677 (1980).

${ }^{10}$ B.I. Lundqvist, Phys. Status Solidi 32, 273 (1969).

${ }^{11}$ J.C. Shelton, Surf. Sci. 44, 305 (1974).

${ }^{12}$ J.C. Ashley and R.H. Ritchie, Phys. Status Solidi B 62, 253 (1974); 83, K159 (1977).

${ }^{13}$ J.C. Ashley, C.J. Tung, and R.H. Ritchie, Surf. Sci. 81, 409 (1979).

${ }^{14}$ C.J. Tung, J.C. Ashley, and R.H. Ritchie, Surf. Sci. 81, 427 (1979)

${ }^{15}$ D.R. Penn, Phys. Rev. B 35, 482 (1987).

${ }^{16}$ J.J. Quinn, Appl. Phys. Lett. 2, 167 (1963).

${ }^{17}$ E. Zarate, P. Apell, and P.M. Echenique, Phys. Rev. B 60, 2326 (1999).

${ }^{18}$ D.R. Penn, S.P. Apell, and S.M. Girvin, Phys. Rev. B 32, 7753 (1985).

${ }^{19}$ H.-J. Drouhin, Phys. Rev. B 56, 14886 (1997).
${ }^{20}$ J. Hong and D.L. Mills, Phys. Rev. B 59, 13840 (1999).

${ }^{21}$ I. Campillo, J.M. Pitarke, A. Rubio, E. Zarate, and P.M. Echenique, Phys. Rev. Lett. 83, 2230 (1999).

${ }^{22}$ W.-D. Schöne, R. Keyling, M. Bandić, and W. Ekardt, Phys. Rev. B 60, 8616 (1999).

${ }^{23}$ I. Campillo, V.M. Silkin, J.M. Pitarke, E.V. Chulkov, A. Rubio, and P.M. Echenique, Phys. Rev. B 61, 13484 (2000).

${ }^{24}$ R. Keyling, W.-D. Schöne, and W. Ekardt, Phys. Rev. B 61, 1670 (2000).

${ }^{25}$ V.P. Zhukov, F. Aryasetiawan, E.V. Chulkov, I.G. de Gurtubay, and P.M. Echenique, Phys. Rev. B 64, 195122 (2001).

${ }^{26}$ V.P. Zhukov, F. Aryasetiawan, E.V. Chulkov, and P.M. Echenique, Phys. Rev. B 65, 115116 (2002).

${ }^{27}$ J.S. Dolado, V.M. Silkin, M.A. Cazalilla, A. Rubio, and P.M. Echenique, Phys. Rev. B 64, 195128 (2001).

${ }^{28}$ A. Fleszar and W. Hanke, Phys. Rev. B 56, 10228 (1997).

${ }^{29}$ A. Fleszar and W. Hanke, Phys. Rev. B 62, 2466 (2000).

${ }^{30}$ E.E. Krasovskii, W. Schattke, V.N. Strocov, and R. Claessen, Phys. Rev. B 66, 235403 (2002).

${ }^{31}$ G.K. Wertheim, D.M. Riffe, N.V. Smith, and P.H. Citrin, Phys. Rev. B 46, 1955 (1992).

${ }^{32}$ L.I. Johansson and B.E. Sernelius, Phys. Rev. B 50, 16817 (1994).

${ }^{33}$ R.A. Bartynski, E. Jensen, T. Gustafsson, and E.W. Plummer, Phys. Rev. B 32, 1921 (1985).

${ }^{34}$ E.V. Chulkov, V.M. Silkin, and E.N. Shirykalov, Surf. Sci. 188, 287 (1987). 
${ }^{35}$ V.M. Silkin and E.V. Chulkov, Fiz. Tverd. Tela. (St. Petersburg) 37, 2795 (1995) [Phys. Solid State 37, 1540 (1995)]; Ph. Hofmann, R. Stumpf, V.M. Silkin, E.V. Chulkov, and E.W. Plummer, Surf. Sci. 355, L278 (1996); T. Balasubramanian, L.I. Johansson, P.-A. Glans, C. Virojanadara, V.M. Silkin, E.V. Chulkov, and P.M. Echenique, Phys. Rev. B 64, 205401 (2001).

${ }^{36}$ L.I. Johansson, H.I.P. Johansson, J.N. Andersen, E. Lundgren, and R. Nyholm, Phys. Rev. Lett. 71, 2453 (1993); E.W. Plummer and J.B. Hannon, Prog. Surf. Sci. 46, 149 (1994); P.T. Sprunger, L. Petersen, E.W. Plummer, E. Laegsgaard, and F. Besenbacher, Science 275, 1764 (1997); T. Balasubramanian, E. Jensen, X.L. Wu, and S.L. Hulbert, Phys. Rev. 57, 6866(R) (1998).

${ }^{37}$ N.W. Ashcroft and N.D. Mermin, Solid State Physics (Saunders College, Philadelphia, 1976).

${ }^{38}$ L. Hedin and B.I. Lundqvist, J. Phys. C 4, 2064 (1971).

${ }^{39}$ V.M. Silkin, E.V. Chulkov, I.Yu. Sklyadneva, and V.E. Panin, Izv. Vuzov. Fiz. 9, 56 (1984) [Sov. Phys. J. 27, 762 (1984)]; E.V. Chulkov, V.M. Silkin, and E.N. Shirykalov, Fiz. Met. Metalloved. 64, 213 (1987) [Phys. Met. Metallogr. 64, 1 (1987)].

${ }^{40}$ V.M. Amonenko, V.Y. Ivanov, G.F. Tikhinskiy, and A. Finkel, Fiz. Met. Metalloved. [Phys. Met. Metallogr. 14, 47 (1962)].

${ }^{41}$ In the present work we use the so-called on-shell approximation (Refs. 42 and 43), when the renormalization constant $Z_{n \mathbf{k}}$ is unity.

${ }^{42}$ L. Hedin and S. Lundqvist, Solid State Phys. 23, 1 (1969).

${ }^{43}$ P.M. Echenique, J.M. Pitarke, E.V. Chulkov, and A. Rubio, Chem. Phys. 251, 1 (2000).

${ }^{44}$ N.E. Maddocks, R.W. Godby, and R.J. Needs, Phys. Rev. B 49, 8502 (1994).

${ }^{45}$ Note that there are two methods to obtain the IMFP, $\lambda(\varepsilon)$, averaged over momenta. The first one is to perform an average of the energy- and momentum-resolved IFMP $\lambda_{n \mathbf{k}}$ over the BZ. In the second one the linewidth $\Gamma_{n \mathbf{k}}$ and the group velocity $\vec{v}_{n \mathbf{k}}$ are separately averaged over the momenta. In the FEG model both methods give identical results. With the use of crystal band structure they should be different. Nevertheless, in the case of $\mathrm{Be}$ the final results are not sensitive to the mode of averaging (in the scale of the presented figures both methods give the same curves). Therefore, for clarity of representation, here we use the second method.

${ }^{46}$ P. Eisenberger, P.M. Platzman, and K.C. Pandy, Phys. Rev. Lett. 31, 311 (1973).

${ }^{47}$ W. Schülke, H. Nagasawa, S. Mourikis, and A. Kaprolat, Phys. Rev. B 40, 12215 (1989).

${ }^{48}$ K.W.-K. Shung, B.E. Sernelius, and G.D. Mahan, Phys. Rev. B 36, 4499 (1987). 